\title{
1 „Die Jagd nach dem Glück“. Zum Erkenntnisinteresse der Untersuchung
}

\author{
„Glück, was ist Glück? Wer weiß es mir zu nennen? \\ Was jeder sucht und was so wen'ge kennen, \\ Wonach wir alle jagen stets und rennen, \\ Wofür selbst Greise glühen noch und brennen, - \\ Glück, was ist Glück? Wer weiß es mir zu nennen? \\ [...] \\ Dem ist es Reichtum, jener nennt es Macht, \\ Dort grünt es einem in des Lorbeers Pracht, \\ Der findet es in wüst durchschwelgter Nacht, \\ Und dieser, wenn es sie beim Buch durchwacht: \\ Glück ist, was jeder sich als Glück gedacht!“1
}

„Glück, was ist Glück? Wer weiß es mir zu nennen?“ Mit dieser Frage eröffnet Reinhold Gerling (1863-1930) das Kapitel seines Glücksratgebers über die „Jagd nach dem Glück“. ${ }^{2} 1920$ ist der Autor davon überzeugt, dass seine arbeitspsychologische Anleitung zur leistungsorientierten Rationalisierung, Disziplinierung und Optimierung die Leserschaft selbstermächtigen könne, ihr als defizitär begriffenes Selbst in eine ,glücklichere' Lebensführung zu transferieren.

Zehn Jahre später, 1930, mitten in der Weltwirtschaftskrise, warnt der Karikaturist Thomas Theodor Heine (1867-1948) ${ }^{3}$, wenige Tage nach dem Wahlsieg der NSDAP an den 5. Reichstagswahlen, vor der „Jagd nach dem Glück“ in einer nationalsozialistischen Lebensführung (Abb. 1) ${ }^{4}$.

Heine illustriert in der ikonografischen Ausgestaltung eines satirischen Kommentars die janusköpfige Wirkungsmacht der griechischen Schicksalsgöttin Fortuna in verfremdeter Gestalt als Adolf Hitler (1889-1945), einem vermeintli-

1 Das Gedicht „Glück“ von Friedrich Halm (1806-1871), eigentlich Eligius Franz Joseph Freiherr von Münch-Bellinghausen, österreichischer Dramatiker, Lyriker, Novellist und Generalintendant des Wiener Hoftheaters, zit. nach Gerling 1920, 170. Vollständiges Gedicht, vgl. Halm 1904, s. Anhang, Kap. 21.1. [Wenn nicht anders vermerkt, sind die Hervorhebungen und Kursivsetzungen in Zitaten dem Original entnommen].

2 Vgl. Gerling 1920, $170-177$.

3 Heine war ein deutscher Karikaturist, Schriftsteller und Mitbegründer des „Simplicissimus“. Nach 1933 sah sich der Antifaschist wegen seines jüdischen Hintergrunds gezwungen, Deutschland zu verlassen und floh ins Exil über Prag, Brünn und Osla nach Stockholm. Zu seiner Person vgl. Hiles 1996.

4 Ausführliche Angaben zu den Bildquellen sind dem Abbildungsverzeichnis (Kap. 20) zu entnehmen.

Ә OpenAccess. (c) 2021 Isabelle Haffter, publiziert von De Gruyter. (cc))BY-NC-ND Dieses Werk ist lizenziert unter einer Creative Commons Namensnennung - Nicht kommerziell - Keine Bearbeitung 4.0 International Lizenz. https://doi.org/10.1515/9783110661439-001 


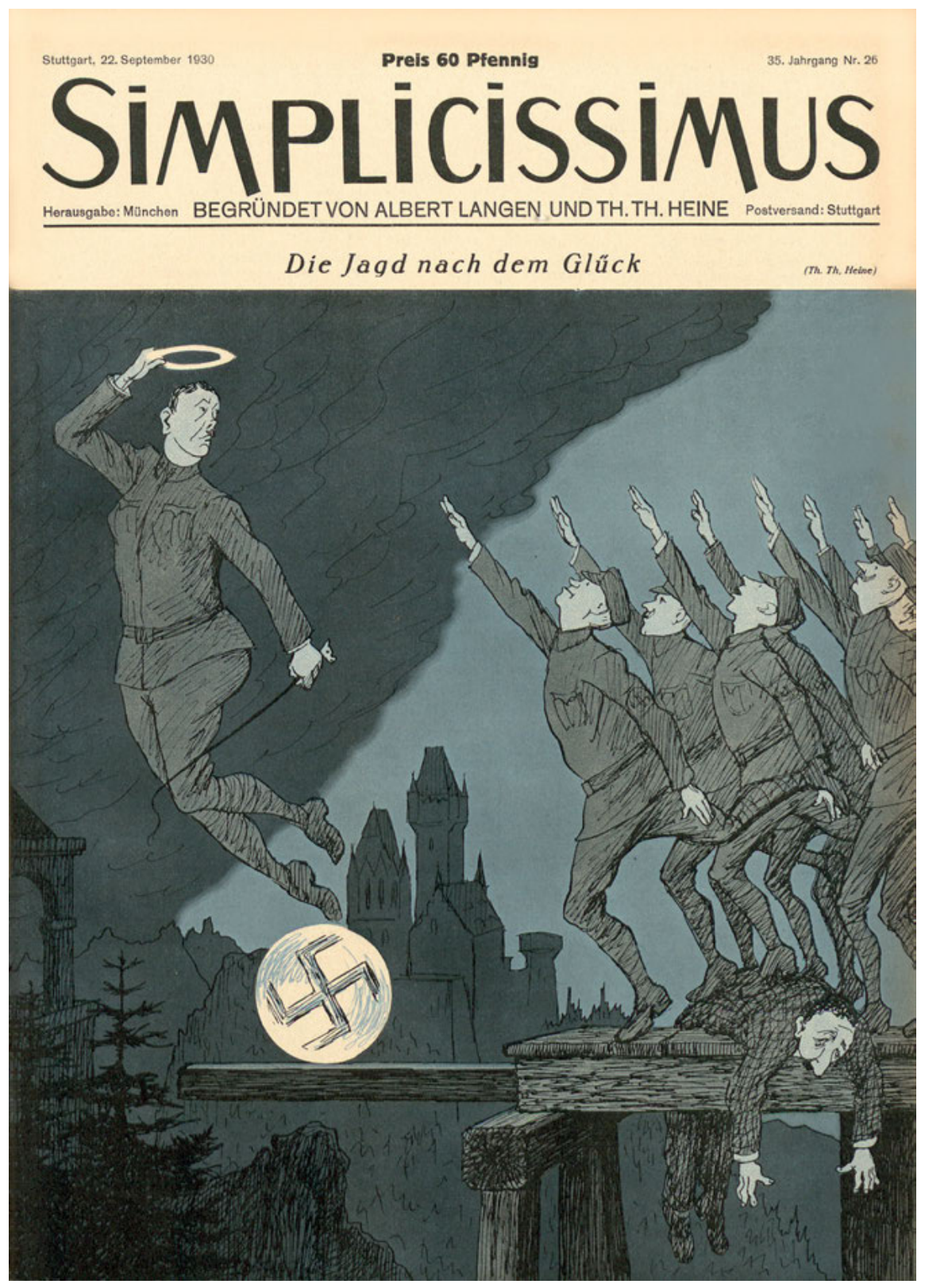

Abb. 1: Thomas Theodor Heine, „Die Jagd nach dem Glück“, Simplicissimus, 1930 
chen Heilsbringer mit Heiligenschein und Peitsche. ${ }^{5}$ Die Vorahnung des Holocausts und des Zweiten Weltkriegs nimmt Heine ikonografisch vorweg: Hitlers antisemitisches Glücksversprechen einer rassistischen Lebensführung werde jüdische Opfer fordern (in der Karikatur stereotypisiert dargestellt) und seine Gefolgschaft auf der „Jagd nach dem Glück“ in den sicheren Tod führen.

Als Vorlage mag das Ölgemälde „Die Jagd nach dem Glück“ (1869) von Rudolf Friedrich August Henneberg (1826-1876) gedient haben (Abb. 2). Darauf jagt die literarische Gestalt des Glücksritters auf hohem Ross dem Trugbild der Fortuna, als Symbol eines irdischen Glücksversprechens, hinterher. Während der Teufel (,das Böse') den Reiter anstachelt und ihm zugleich als Allegorie des Todes im Nacken sitzt, fordert der Reiter sein Schicksal auf Kosten seines Lebens und des Lebens anderer heraus. Auf seiner ,Jagd nach dem Glück“ fällt die Allegorie der Tugend und Moral (,das Gute`) dem Getriebenen zum Opfer.

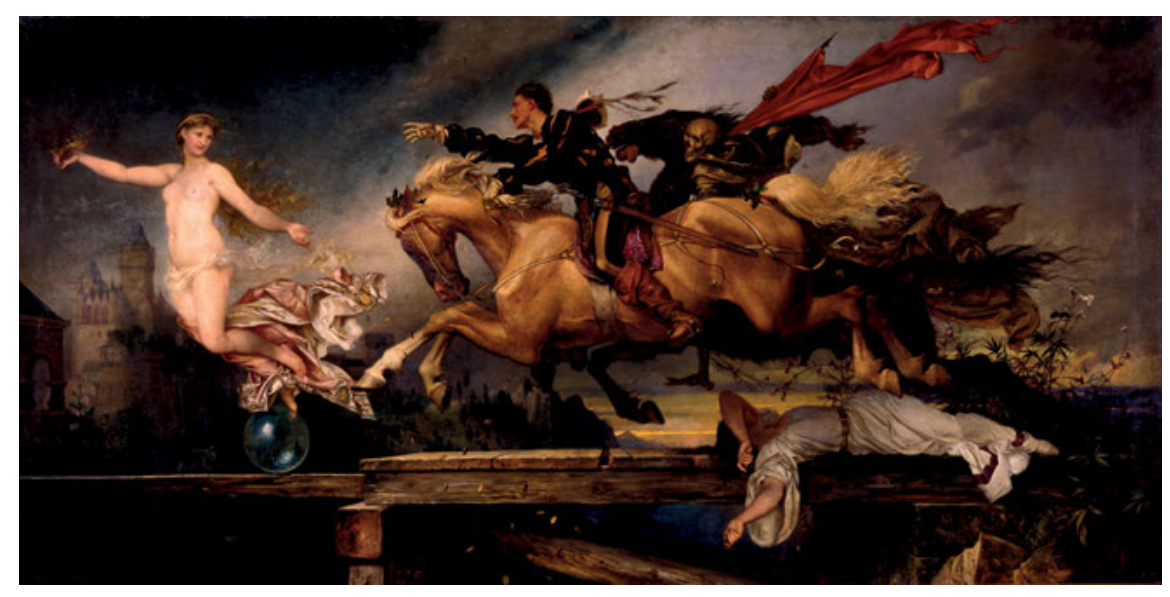

Abb. 2: Rudolf Friedrich August Henneberg, „Die Jagd nach dem Glück“, 1863-1868

Die exemplarische Gegenüberstellung unterschiedlicher Vorstellungen von der „Jagd nach dem Glück“ macht das Erkenntnisinteresse der vorliegenden Untersuchung deutlich: „Glück“ ist in erster Linie ein Quellenbegriff, der als historischer Forschungsgegenstand einer Wissensgeschichte von Brüchen und Kontinuitäten geprägt und selbst von komplexer Ambivalenz gekennzeichnet ist.

5 Zur kunsthistorischen Methodik der Bildanalyse s. Kap. 4.1. 


\subsection{Ausgangspunkt der Untersuchung}

Den Ausgangpunkt der Untersuchung bildet die quellengestützte Feststellung, dass im Untersuchungszeitraum von 1933 bis 1945 im transnationalen Vergleich zwischen NS-Deutschland und der Schweiz eine dezidiert affirmative Gefühlspolitik nachweisbar ist, die sich aus einer wissenshistorischen Perspektive als eine Politik der ,Glückskulturen“ untersuchen lässt. Diese quellenbasierte Erkenntnis mag zunächst erstaunen. Historische Untersuchungen über den Nationalsozialismus legten ihren Fokus lange Zeit auf die aggressive Propagandastrategie der NSDAP, welche auf einer antisemitischen und antibolschewistischen Gefühlspolitik basierte. ${ }^{6}$ Diese Forschungsansätze konzentrierten sich auf die historische Analyse negativer Feindbilder und Gefühlsbeschreibungen (Hass, Neid, Demütigung oder Verachtung), welche im Rahmen der antisemitischen Hetze, Verfolgung und des Holocausts propagiert wurden. ${ }^{7}$ Seit einigen Jahren legen jedoch Historiker^innen wie Ute Frevert („Gefühlspolitik“), Ian Kershaw („Der Hitler-Mythos“) und Peter Reichel („Schöner Schein des Dritten Reiches“) ihr Augenmerk auch auf affirmative Gefühlspolitiken.

Die vorliegende Untersuchung knüpft an letzteren Forschungszweig an und erweitert diesen um ein Forschungsdesiderat: Eine Wissensgeschichte über eine Politik transnationaler Wissens- und Gefühlskulturen eines propagierten „Glücks“.

\subsection{Thesen und Fragestellungen}

Wissen über „Glück“ bedeutet Macht. Zur Selbstdarstellung einer Nation, ihres Werte- und Moralsystems, kann auf Glückswissen ${ }^{8}$ zurückgegriffen und dieses als emotionspolitisches Machtinstrument eingesetzt werden. ${ }^{9}$ Die vorliegende Untersuchung möchte aus einer wissenshistorischen Perspektive heraus aufzeigen, wie eine Politik ${ }^{10}$ der ,Glückskulturen' in NS-Deutschland als eine rassistische

6 Zum Forschungsstand s. Kap. 2.1.

7 Zum aktuellen Stand der NS-Forschung vgl. Echternkamp 2018, insb. 135-254.

8 Zum Begriff vgl. Stephanie Kleiners Forschungsprojekt „Glückswissen. Zur Geschichte einer Reflexionsform gelingender Integration im 20. Jahrhundert“ (2011-), Exzellenzcluster Kulturelle Grundlagen von Integration, Universität Konstanz, https://www.exzellenzcluster.uni-konstanz.de/2724.html, 09.01.2020, vgl. Kleiner 2011, Kleiner 2014, Kleiner und Suter 2015, Kleiner 2015, Kleiner 2016.

9 Zum Aktualitätsbezug s. später in diesem Kapitel und im Ausblick (Kap. 18).

10 Mehr zum Begriff des „Politischen“ s. Kap. 1.4. 
Arbeitsmoral (mit der Parole „Kraft durch Freude“) und zeitgleich in der Schweiz als eine kulturnationalistische Gefühlspolitik (der sogenannten „geistigen Landesverteidigung“'11) in Erscheinung trat.

Die Untersuchung legt den Fokus auf die Umstände und Machtverhältnisse, in denen eine Politik der ,Glückskulturen' als solche wahrgenommen und beschrieben, als Wissensbestand rezipiert und umgedeutet sowie als Wissenspraktik ausgeübt, verändert oder verboten wurde.

Die zentralen Forschungsfragen lauten:

1. Wie veränderte sich Glückswissen im Übergang von der Weimarer Republik zum Nationalsozialismus im transnationalen Vergleich zur Schweiz?

2. Wo sind Brüche, Kontinuitäten und Ambivalenzen festzustellen?

Den Untersuchungsgegenstand bildet die transnationale Wissenszirkulation von ,Glückskulturen' in den Bereichen Politik, Wissenschaft und der darstellenden Künste. Um den Untersuchungsgegenstand einzugrenzen, liegt das Augenmerk auf der länderspezifischen Arbeits- und Theaterpolitik, den transnational rezipierten Wissensdisziplinen der Arbeitspsychologie und der Theaterwissenschaft sowie der transnational ausgeübten Wissenspraktik der Ratgeber- und Theaterkulturpraxis.

Die Wahl des Untersuchungsgegenstands ist wie folgt begründet: Bis heute befassen sich die Wissenspraktiken der Arbeitspsychologie und Theaterwissenschaft mit Gefühlen und deren Wirkung und Rezeption, sei es in empirischen Forschungen zum leistungsorientierten Arbeitsglück oder zu theaterästhetischen Gemeinschaftserfahrungen. Für den Untersuchungszeitraum kann nachgewiesen werden, dass sowohl die Arbeitspsychologie als auch die Theaterwissenschaft daran beteiligt waren, Wissen über „Glück“ zu erzeugen, zu verbreiten und zu verändern und nach 1933 in den politischen Dienst ihrer Nation zu stellen. Dabei fand eine transnationale Wissenszirkulation zwischen den Wissensdisziplinen und den jeweiligen Wissenspraktiken statt. Die Untersuchung fragt folglich nach

11 Der Begriff „geistige Landesverteidigung“ (GV) war seit Ende der 1920er Jahre Bestandteil der nationalistischen Diskurse in rechts-konservativen Kreisen und konnte bis Mitte der 1930er Jahre im politischen Bewusstsein der breiten Schweizer Öffentlichkeit Fuß fassen. Doch erst mit der bundesrätlichen Botschaft ,über die Organisation und die Aufgaben der schweizerischen Kulturwahrung und Kulturwerbung “vom 9. Dezember 1938 (vgl. Botschaft des Bundesrats 1938, 985), die im Wesentlichen vom rechts-konservativen, katholischen Bundesrat Philipp Etter verfasst worden war, konnte die GV als propagandistische Kulturpolitik ideologisierend und mythologisierend wirksam werden. Mehr zum Begriff s. Kap. 15.5, vgl. Mooser 1997, vgl. Tanner 1998, vgl. Kreis 2011, vgl. Jorio 2006, Tanner 2015. 
den transnationalen „Denkstilen“12 (Mannheim) und nach den rivalisierenden „Denkkollektiven“13 (Fleck) innerhalb der Arbeitspsychologie und der Theaterwissenschaft. Kulturelle Wissenspraktiken, wie z.B. die auf arbeitspsychologischen Erkenntnissen basierende Ratgeberpraktik oder die theaterwissenschaftlich erforschte Laientheaterpraxis, werden als Teil der Politik von ,Glückskulturen analysiert.

Eine Wissensgeschichte, die „Glück“ als ihren Untersuchungsgegenstand bezeichnet, geht davon aus, dass gesellschaftliche Vorstellungen von „Glück“ einem komplexen historischen Bedeutungswandel unterworfen sind. Die Wissensproduktion von „Glück“ und dessen Trabanten (Freude, Frohsinn, Euphorie) stellt ein Wissen aus unterschiedlichen Wissensdisziplinen dar, welches sich in einem kontinuierlichen Zirkulationsaustausch zwischen Expertenwissen (Bsp. Arbeitspsychologie) und Populärwissen (Bsp. Glücks- und Erfolgsratgeberliteratur) befindet. Die Untersuchung richtet folglich ihren Fokus auf die historische Konstruiertheit des Wissens über „Glück“. Sie fragt beispielsweise, inwiefern sich emotionspolitische Moralvorstellungen von „Glück“ in kulturnationalistischen Praktiken, wie der nationalen Festspielgestaltung, niederschlugen. Welchen Beitrag leistete die Glückserfahrung der Laienspieler`innen für die Wissens(re)produktion einer „völkerpsychologischen“ Theoretisierung in der Theaterwissenschaft?

Zur Beantwortung dieser Fragen liegen der Untersuchung folgende Prämissen zugrunde:

1. Aussagen über „Glück“, das sogenannte Glückswissen, werden als „Teil des kulturellen Wissens ${ }^{\text {“14 }}$ verstanden.

2. Brüche, Kontinuitäten und Ambivalenzen von Glückswissen lassen sich in aufeinander bezogenen Wechselwirkungen aufzeigen. ${ }^{15}$

3. Glückswissen lässt sich auf einer zeitlichen, geografischen und politischen Achse analysieren. Die zeitliche Achse fokussiert die Jahre zwischen 1933 und 1945, die geografische Achse NS-Deutschland und die Schweiz. Auf der politischen Achse wird, ausgehend vom Nationalsozialismus, in einem transnationalen Vergleich die Schweizer Politik der „geistigen Landesverteidigung" untersucht, die noch bis in die 1970er Jahre nachwirkte.

12 Vgl. Mannheim 2015, 5; s. Kap. 9.7.

13 Vgl. Fleck 1983; s. Kap. 9.7, 10.4., 11.1., 11.2, 15.7.

14 Müller-Wille, Reinhardt, Sommer 2017, 4.

15 Einen Denkanstoß zu dieser These gab u. a. der Sammelband von Birthe Kundrus und Sybille Steinacher, vgl. Kundrus und Steinbacher 2013. 
4. Gefühls- und Wissenskulturen von „Glück“, kurz, Glückskulturen ${ }^{\text {‘16 }}$, schlagen sich in soziokulturellen Praktiken, wie der Ratgeberwissenspraktik oder der Laienspielpraxis, nieder und setzen sich aus einer Vielzahl unterschiedlicher Akteur*innen, Institutionen und Netzwerken zusammen, die ihrerseits an der transnationalen Wissensproduktion teilhaben. ${ }^{17}$,Glückskulturen ${ }^{6}$ und Glückswissen sind gleichermaßen von Brüchen, Kontinuitäten und Ambivalenzen gekennzeichnet und können folglich auf einer zeitlichen, geografischen und politischen Achse wissenshistorisch analysiert werden.

Trotz des transnationalen Ansatzes ${ }^{18}$ muss eine historische Forschung zum Untersuchungszeitraum 1933-1945 die beispiellosen Gewaltexzesse im Rahmen der nationalsozialistischen „Rassenpolitik“ betonen: Die affirmative Gefühlskultur der NS-Arbeitsmoral „Kraft durch Freude“ führte die „NS-Volksgemeinschaft“ in den Zweiten Weltkrieg. Rund 6 Millionen europäische Jüd*innen kamen während des nationalsozialistischen Völkermords (Schoah, hebr. „Katastrophe“, oder Holocaust, griech. „vollständig verbrannt“) ums Leben. Für die historische Forschung, die sich mit dem Nationalsozialismus auseinandersetzt, sind laut Jörg Echternkamp drei grundlegende Fragen von zentraler Bedeutung:

16 „Glückskultur“ im Singular wurde gemäß dem Wissensstand der Autorin erstmals 1916 vom Anthroposophen Rudolf Steiner (1861-1935) in Bezug auf die Atlantis-Legende zur Schaffung einer „Glückskultur“ durch die Kraft Ahrimans (dem Gegenspieler von „Luzifer“) verwendet, vgl. Steiner 1984, 113. Steiner beschrieb in seinem Vortrag, wie unter „ahrimanischem Einfluss“ eine seit dem „fünften nachatlantischen Zeitalter“ (15. Jh. n.u.Z. bis ins 4. Jahrtausend) wirkende Kultur erschaffen worden sei, welche „das Gute im Glücke suchte“ und als Endzweck das Erlangen „menschlichen Glück[s]“ vorsehe, womit „das Glück selber als das Wünschenswerte“ betrachtet würde. Steiners kulturkritischer Vortrag, der neben theosophischen auch implizit psychoanalytische Bezüge auf Freuds Trieb- und Lusttheorie aufweist, kritisierte im Wesentlichen den Utilitarismus und das Versprechen des Staates auf „Das größte Glück der größsten Zahl“ (Jeremy Bentham), vgl. Steiner 1984, 109. Dieser „Glückskultur“ stellte Steiner sein anthroposophisches Gesellschaftsmodell entgegen, vgl. Steiner 1984, zu „Glück“ und „Glückskulturen“ 108-113, 108 und 113. Der promovierte Philosoph, Publizist und Esoteriker Steiner gründete 1913 die „Anthroposophische Gesellschaft“ in Berlin und 1923 die „Allgemeine Anthroposophische Gesellschaft“, deren Sitz bis heute im „Goetheanum“ in Dornach bei Basel ist. Steiner war u.a. Begründer der Bewegungslehre „Eurythmie“ (1913), der „Heilpädagogik“ (1924), der biologischdynamischen Landwirtschaft „Demeter“ (1924) sowie der „Rudolf-Steiner-Schule“ („Waldorfschule“). Die ersten Schulen entstanden 1919 in Stuttgart und 1926 in Basel (CH). Zu seiner Person vgl. Höhne 2012. Mehr zu Glücksratgebern und -vorstellungen s. Kap. 8-14.

17 Vgl. Müller-Wille, Reinhardt, Sommer 2017, 10.

18 Zum transnationalen Ansatz s. Kap. 4. 
1. Wie war es möglich, dass die nationalsozialistische Bewegung aus der demokratischen Weimarer Republik heraus nach 1933 eine Diktatur errichten und stabilisieren konnte?

2. Wie ließ sich die Mehrheit der politisch heterogenen und vom Ersten Weltkrieg traumatisierten Bevölkerung Deutschlands in die sogenannte „Volksgemeinschaft" einbinden?

3. Wie ist eine mehrheitsgesellschaftliche Mobilisierung für einen beispiellosen Vernichtungskrieg und den Holocaust auf der Grundlage der „NS-Rassenideologie“ zu erklären? ${ }^{19}$

Die vorliegende Untersuchung erhebt nicht den Anspruch, abschließende Antworten auf die komplexen Fragen der aktuellen NS-Forschung geben zu können. Dennoch verortet sie sich im wissenschaftlichen Diskursfeld der historischen Forschung zum Nationalsozialismus, indem sie eine Politik von ,Glückskulturen in NS-Deutschland und in der Schweiz zwischen 1933 und 1945 transnational untersucht.

\subsection{Was bedeutet „Glück“? Oder: Was „Glück“ bedeuten kann}

Zur Beantwortung dieser Frage bedarf es einer Begriffserläuterung. Die Untersuchung fasst Glück weder als ein eindeutig bestimmbares Gefühl ${ }^{20}$ noch als einen abschließend definierbaren Wissensbestand ${ }^{21}$ auf. „Glück“ als ein historischer Untersuchungsgegenstand der interdisziplinären Kulturwissenschaften wird als vielschichtiger, dynamischer und daher im höchsten Maße ambivalenter Quellenbegriff verstanden.

Was kann „Glück“ im Untersuchungszeitraum 1933-1945 bedeuten?

Am 11. Dezember 1935 hielt der „Reichsleiter“ der „Deutschen Arbeitsfront“ (DAF) und Mitbegründer der Freizeitorganisation „Kraft durch Freude“ (KdF), Dr. Robert Ley (1890 - 1945), an einer Großkundgebung vor rund 20.000 „schaffenden deutschen Menschen“ eine Rede über das Ziel der „Deutschen Arbeitsfront“:

19 Zum aktuellen Stand der NS-Forschung vgl. Grundprobleme und Tendenzen. In: Echternkamp 2018, insb. 135-254.

20 „Gefühl“ und „Emotion“ werden im Folgenden synonym verwendet, vgl. Was ist Emotion? In: Plamper 2012a, 20 - 34. Mehr zur Gefühlsgeschichte s. Kap. 2.1.

21 Mehr zur Wissensgeschichte s. Kap. 2.1. 
„Wir haben heute ein stattliches Fundament gebaut. Wir können weiterbauen. Jeden Tag kommen neue Fanatiker ${ }^{22}$, neue Glaubensbekenner zu uns. Auch sie erklären: Hitler hat immer recht! Wir wissen, wozu wir leben. Wir begreifen und wir verstehen, was Glück ist. Wir bekennen uns zum Nationalsozialismus. Der Erfolg wird immer größer. Deutschland wird immer schöner werden, und dieses Volk wird immer glücklicher sein. [...] So geht es weiter von Geschlecht zu Geschlecht. Deutschland wird schöner und glücklicher werden. [...].“23

Der „Reichsleiter“ schien 1935 genau zu ,wissen“, was „Glück“ bedeutet: Es sei Hitlers „Rassenideologie“ einer nationalsozialistischen „Volksgemeinschaft“ als selbstdisziplinarischer „Volkskörper“ konzipiert, der sich selbst mittels einer leistungsorientierten Arbeitsmoral, der sogenannten „Kraft durch Freude“, ein autosuggestiv-kollektivistisches „Glück“ bescheren könne. ${ }^{24}$ „Kraft durch Freude“ definierte Ley explizit als Politik einer nationalsozialistischen, Glückskultur‘:

„[...] weder Gewaltmaßnahmen noch Gesetze können das Glück der Menschen begründen, sondern nur solche Organisationen, die der seelischen und völkischen Haltung eines Volkes entsprechen und dadurch seine lebensbejahenden Kräfte zur Wirkung bringen. ,Kraft durch Freude“ ist die große Gemeinschaft, in der die aus dem tiefsten Wesen des deutschen Volkes kommende Lebenskraft und Lebensfreude wirkt und lebt [...].“25

Ein Blick in die Gegenwart zeigt, dass auch heute noch affirmative Gefühlspolitiken in transnationalen Gesellschaftsbereichen propagiert werden. Eine Wissenskontinuität ist beispielsweise in der neueren Glücksforschung zu verzeichnen, welche die politische Komponente von Glückswissen und die Bedingungen einer Gesellschaft und jeder Einzelperson zueinander in Beziehung stellt. Damit ist nicht gemeint, dass Nationalsozialismus und Neoliberalismus gleichzusetzen sind. Eva Illouz und Edgar Cabanas bezeichnen den „sozialen Imperativ“ eines

22 „Fanatiker“ ist bei Ley ein positiv konnotierter Charakterzug, mit welchem er in seiner Rede auch die „Hitler-Jugend“ und „Werkscharen“ bezeichnet, die den Nationalsozialismus im Sinne Leys weiter ausbauen würden: „Die werden weiterbauen. Sie sind noch größere Fanatiker als wir. Die haben noch weniger Vorurteile als wir und brauchen nicht mit alten Dingen zu ringen. Die kommen gleich aus dieser neuen Welt, die sind noch glaubenskräftiger als wir, sind unduldsamer, die dulden nichts mehr, die räumen augenblicklich auf, wo wir noch zaghaft sind.“ Ley 1937a, 207. 23 Ebd.

24 Einen Anschluss an diese These findet man in der aktuellsten Geschichtsforschung, u.a. Föllmer 2020, Frevert 2019a, 2019b, Wildt 2019, Frei 2018, Süß 2018, Kershaw 2014 sowie im Forschungsprojekt von Ute Frevert „Die Macht der Gefühle. Politische Kommunikation zwischen unten und oben“, MPIB, Forschungsbereich „Geschichte der Gefühle“, Berlin, https://www.mpibberlin.mpg.de/forschung/forschungsbereiche/geschichte-der-gefuehle/forschungsfelder/buer ger-und-nation/die-macht-der-gefuehle, 12.01.2019.

25 Ley 1937b. Vollständige Definition von „Kraft durch Freude“ s. Anhang, Kap. 21.2. 
selbst erlernbaren Glückswissens als „das Glücksdiktat“ unserer Zeit. ${ }^{26}$ „Glück ist ein Markenzeichen moderner Moral und steht im Mittelpunkt von Politik“, äußerte Illouz in einem SPIEGEL-Interview. ${ }^{27}$ Dieses Wissen zirkuliere in Glücksseminaren, Glücksratgeberbüchern, Schulfächern, Social Media und computertechnischen Apps. Insbesondere im Bereich der „Wissenschaft des Glücks“28 problematisieren die Soziolog^innen die politische und wirtschaftliche Instrumentalisierung von „Glück“ und die gesellschaftliche Wechselwirkung. Sie fragen, wer sind die Gewinner`innen und wer die Verlierer`innen der „Diktatur des Glücks“? Wer trägt zuletzt die Verantwortung für das Erlangen eines „Glücks“ in einem neoliberalen System: die Politik oder jede Einzelperson für sich selbst?

Dabei beziehen sich die Autor*innen auf die wirtschaftsorientierte Positive Psychology, wie sie seit rund 20 Jahren vom US-amerikanischen Psychologen Martin Seligman ( $\left.{ }^{\star} 1942\right)$ prominent vertreten wird. Das Programm „Comprehensive Soldier Fitness“ (CSF) der US-Armee, das in Zusammenarbeit mit Seligman seit 2008 zur emotionspolitischen Rationalisierung der US-Armee entwickelt wurde, zeige exemplarisch, wie sich die arbeitspsychologische Positive Psychology „,in den Dienst so ziemlich jeder Institution [stellt], die ein Interesse daran hat, die Stimmung und das Verhalten von Menschen zu kontrollieren. Militär, Unternehmen, Staaten.“29

Im Kontext eines zunehmend globalen Neoliberalismus führten politisch initiierte, internationale Vergleichsstudien, wie der „World Happiness Report“ ${ }^{\star 30}$ mit seinen empirisch erhobenen Glücksindizes, zur politischen und wirtschaftlichen Instrumentalisierung der Glückswissenschaft. Illouz und Cabanas zufolge zeichne sich eine bedenkliche gesellschaftliche Entwicklung ab: „Es ist viel ein-

26 Vgl. Cabanas und Illouz 2019a.

27 Vgl. Illouz 2019, 105.

28 Vgl. ebd.

29 Vgl. ebd.

30 Mit Hilfe des Expertenwissens aus der ökonomischen Glücksforschung versuchen 156 Regierungen anhand des jährlich publizierten „World Happiness Reports“ das „Glück“ bzw. die „life satisfaction“ ihrer Bevölkerung indexikalisch zu messen, um es sozialwirtschaftlich optimieren zu können. Dabei gilt es, das Kleingedruckte auf Seite 3 zu beachten: „Any views expressed in this report do not necessarily reflect the views of any organisation, agency or program of the United Nations“ Helliwell, Layard und Sachs 2019, 3. Seit 2012 listet der World Happiness Report 156 Länder nach ihrem Glücksindex „to inform their policy-making decisions.“ 2017 wurden erstmals auch Teile Afrikas und Chinas miteinbezogen. Rangliste 2019: 1. Finnland, 2. Dänemark, 3. Norwegen, 4. Island, 5. Holland, 6. Schweiz, 10. Österreich, 17. Deutschland, vgl. Helliwell, Layard und Sachs 2019. Kritische Studien zur Glücksforschung und aktuellen Glücksratgeberliteratur vgl. Duttweiler 2007, Ahmed 2010, Glück als Ziel der Politik? Neue Argumente für eine Gefühlspolitik. In: Heidenreich und Schaal 2012, 217-234, David 2014, Nay 2014, Kleiner 2015, Kleiner 2016, Freitas 2017, Cabanas und Illouz 2018. 
facher, die individuellen Glücksgefühle $\mathrm{zu}$ manipulieren, als die Struktur von Eigentum oder Ungleichheit zu ändern“, so Illouz über die politische Instrumentalisierbarkeit von Glücksindizes auf Kosten sozialer Gleichheit und Gerechtigkeit. $^{31}$

Als Ergänzung zur wirtschaftspsychologischen Glückswissenschaft muss neben der neurologischen insbesondere die genetische Glücksforschung erwähnt werden. Diese verfolgt den biologistischen Ansatz, dass „Glück“ genetisch über ein sogenanntes Glücksgen (5-HTTLPR) erzeugbar sei. ${ }^{32}$ Den Ausgang dieser Entwicklung gilt es ebenfalls abzuwarten.

Die vorliegende Arbeit nimmt sich der bis in die Gegenwart nachweisbaren Ambivalenz und Kontinuität von Glückswissen und deren politischer Instrumentalisierbarkeit mit Blick auf den Untersuchungszeitraum 1933-1945 an. Die Untersuchung widmet sich der affirmativen Gefühlspolitik, die als ,Volksglück nicht zwangsläufig das „Glück“ der Einzelperson miteinschließt.

\subsection{Politik der ,Glückskulturen‘: Eine Begriffserläuterung}

Eine grundlegende These der vorliegenden Untersuchung lautet, dass Glückswissen als Teil einer affirmativen Gefühlspolitik, mit der Zäsur von 1933, einen historischen Bedeutungswandel durchlief, der von Brüchen, Kontinuitäten und Ambivalenzen geprägt ist. Was ist mit affirmativen Gefühlen gemeint? Im Sinne einer Gefühlsgeschichte, wie sie Ute Frevert vertritt, werden Gefühle als historisch geformter, kulturell erlernter, daher zeitlich und örtlich spezifischer Teil des kulturellen Wissens von hoher ambivalenter Komplexität begriffen. Gefühle können, laut Frevert, in unterschiedlichen multimedialen Mischformen (Worten, Bildern, Gesten, Mimik, Klängen) und als „moralische Ökonomien“ („moral economies“)”33 dynamisch auf Individuen, soziale Interaktionen und Praktiken einwirken und selbst zum Diskursgegenstand werden. ${ }^{34}$

Zur historischen Erforschung von Gefühlspolitiken unterscheidet Frevert zwischen einer ,gefühlspolitische[n] Adressierung (durch das Regime und re-

31 Vgl. Illouz 2019, 105.

32 Vgl. u.a. Neve 2011. Zur genetischen Forschung als ,moderne‘ Dienstleistung vgl. Sommer 2016.

33 Vgl. Frevert 2019c. Der Sozialhistoriker Edward P. Thompson prägte den Begriff 1971 zur Untersuchung der Verhältnisse zwischen moralischen Werten und Wirtschaftssystemen im 18. Jahrhundert. Vgl. Thompson 1971. Zur Gefühlsgeschichte und „moral ecomonies“ s. Kap. 2.1. 34 Vgl. Frevert 2009, 191 f. Vgl. Laukötter [o. J.], Hitzer 2011. Mehr zur Gefühlsgeschichte s. Kap. 2.1. 
gimetreue Medien) und Gefühlen als sozialer Praxis“. ${ }^{35}$ Diese analytische Trennung ermöglicht es, zwischen „verordneten Gefühlen“ und jenen Gefühlen zu unterscheiden, welche beispielsweise in der DDR die Teilnehmer*innen an Pfingst- und Jugendtreffen tatsächlich empfanden, ,vermittelt und transportiert durch gemeinsames Singen, Marschieren, Skandieren“ “. ${ }^{36}$

Gefühle, welche im Zusammenhang mit einem Konglomerat von Nationalgefühlen („Glück“, Ehre, Stolz, Verbundenheit, Opferbereitschaft, Freiheit, Heimatliebe) stehen und kulturnationalistische Moralvorstellungen und ein nationales Machtsystem bejahen, werden in der vorliegenden Untersuchung als affirmative Gefühle beschrieben, die sowohl top down propagiert als auch bottom up von der Bevölkerung empfunden werden konnten. Politisch instrumentalisiertes Wissen über „Glück ${ }^{\text {“37 }}$, welches als affirmative Nationalgefühle eines kulturnationalistischen Moral- und Machtsystems in den Bereichen Politik, Wissenschaft und der darstellenden Künste in den Quellen nachweisbar ist, wird als Teil einer affirmativen Gefühlspolitik begriffen und aus einer wissenshistorischen Perspektive als soziokulturelle Praktik einer Politik von ,Glückskulturen ${ }^{68}$ untersucht.

Ein Beispiel: Gefühle wie „Glück“, „Liebe“ oder „Treue“ sollten im NS-Regime als Mittel gefühlspolitischer Instrumentalisierung die Bevölkerung emotional ergreifen und selbsterzieherisch bzw. selbstdisziplinarisch mobilisieren. ${ }^{39}$ Schließlich war sich Hitler der psychologisch wirkungsmächtigen Illusion der emotionspolitischen Propaganda bewusst, wie das Kapitel „Die Aufgabe der Propaganda“ aus „Mein Kampf“ zeigt: „[...] so muß [sic] ihr Wirken [der Propaganda] auch immer mehr auf das Gefühl gerichtet sein und nur sehr bedingt auf den sogenannten Verstand.“40

35 Zu Gefühlsstilen, Gefühlspraktiken, Gefühlspolitik vgl. Frevert 2020b. Hier vgl. Göhlsdorf 2020, 2.

36 Vgl. Göhlsdorf 2020, 2. Mehr zur affirmativen Gefühlspolitik in der DDR vgl. Brauer 2020.

37 Zur Etymologie s. Kap. 2.2.

38 Zur wissenshistorischen Bedeutung des Begriffs „Glückskultur“ bei Rudolf Steiner s. Kap. 1.2. 39 Mehr zur Selbsterziehung und Selbstdisziplinierung s. u.a. Kap. 7-14. Frevert legt in ihrem Aufsatz zur Gefühlspolitik im NS-Regime den Fokus auf Gefühle wie Liebe, Treue und Hass, wobei sie „Glück“, „Freude“ und „Euphorie“ erwähnt, aber nicht ins Zentrum ihrer Analyse stellt. Vgl. Frevert 2015.

40 Vgl. Hitler 2016a, 499. Auf Hitlers Gefühlspolitik und dessen wissenshistorische Kontexte aus dem Bereich der Massenpsychologie (u. a. bei Gustav Le Bon) und der Arbeitswissenschaft wird in Kap. 15.6 ausführlich eingegangen. [\#-Zeichen sind der kritischen Edition entnommen und weisen auf abweichende Formulierungen, je nach Auflage von „Mein Kampf“, hin]. 
„Die Aufgabe der Propaganda liegt nicht in einer wissenschaftlichen Ausbildung des einzelnen, als vielmehr\# in einem Hinweise\# der Masse auf bestimmte Tatsachen, Vorgänge, Notwendigkeiten usw. deren Bedeutung dadurch erst in den Gesichtskreis\# der Masse gerückt werden soll.

Die Kunst liegt nun ausschließlich darin, dies in so vorzüglicher Weise anzufassen\#, daß eine allgemeine Überzeugung von der Wirklichkeit einer Tatsache, der Notwendigkeit eines Vorganges, der Richtigkeit von etwas Notwendigem usw. entsteht. Da sie nun nicht Wissenschaft\# an sich ist und sein kann, da ihre Aufgabe ja genau wie bei dem Plakat im Aufmerksammachen der Menge zu bestehen hat [...], so muß [sic] ihr Wirken auch immer mehr auf das Gefühl gerichtet sein und nur sehr bedingt auf den sogenannten Verstand.

Jede Propaganda hat volkstümlich zu sein und ihr geistiges Niveau zu richten\# nach der Aufnahmefähigkeit des Beschränktesten unter denen, an die sie sich zu richten gedenkt. [...]

Je bescheidener dann ihr wissenschaftlicher Ballast ist, und je mehr sie ausschließlich auf das Fühlen der Masse Rücksicht nimmt, um so durchschlagender wird\# der Erfolg sein\#." ${ }^{41}$

Entgegen dem von der NS-Partei propagierten „Führer“-Kult eines fürsorglichen Vaters der „Volksgemeinschaft“, äußert Hitler in „Mein Kampf“ seine zutiefst verächtliche Haltung gegenüber der „Masse“ und deren angeblich „Beschränktesten“. Die Intelligenz der „Masse“ marginalisierend, war Hitlers Propaganda „volkstümlich“. Sie war ein antiintellektuelles Instrument zur politischen Selbstdarstellung, emotionspolitischen Emotionalisierung und autosuggestiven Mobilisierung, das jedoch auf wissenschaftlichen Erkenntnissen, u. a. aus dem Bereich der Arbeitspsychologie und Theaterwissenschaft, aufbaute, wie die Untersuchung zeigen wird..$^{42}$ Das Ziel der Propaganda sei ein emotionspolitisches „Aufmerksammachen der Menge“, ähnlich der Reklame auf Werbeplakaten, so Hitler. Nicht die ,Wahrheit' sei von Belangen. Zentral sei, wie in der Werbung, die ,allgemeine Überzeugung von der Wirklichkeit einer Tatsache, der Notwendigkeit eines Vorgangs [...]“. ${ }^{43}$ Die propagandistische Gefühlspolitik sei, frei von wissenschaftlichen Verifizierungsnöten, allein „auf das Fühlen der Masse“ ausgerichtet. Darin

41 Hitler 2016b, 499. Auf das theaterwissenschaftliche und -ästhetische Wissen Hitlers wird bspw. in Kap. 15.6 Bezug genommen.

42 Vgl. Hitler 2016b, 499. Die massenpsychologische Forschung, u. a. bei Gustav Le Bons „Psychologie des Foules“ (1895), wurde sowohl von Hitler als auch Mussolini rezipiert. Daneben beeinflusste sie die Psychoanalyse Freuds (u.a. „Massenpsychologie und Ich-Analyse“) oder die soziologische Forschung Webers und Simmels, vgl. Moscovici 1984, 89-93. Wilhelm Reich wandte 1933 den freudschen Ansatz an, um die nationalsozialistische Massenpsychologie zu erforschen, vgl. Das nationalsozialistische Selbstgefühl. In: Reich 2011, 75-77. Mehr dazu s. Kap. 10, 11, 15.5, 15.6.

43 Vgl. Hitler 2016b, 499. Zum Aktualitätsbezug s. Kap. 18. 
läge ihr massenpsychologisches Potential für einen „durchschlagende[n] [...] Erfolg. “44

Ausgehend von Hitlers Definition der nationalsozialistischen Propaganda als ein massenpsychologisches Instrument einer affirmativen Gefühlspolitik, sind für eine wissenshistorische Untersuchung einer Politik von ,Glückskulturen ${ }^{6}$ drei Prämissen vorausgesetzt, welche den Begriffen „Kultur“, „Wissen“ und „Politik“ zugrunde liegen:

1. Kulturwissenschaften bezeichnen Kultur als ihren Untersuchungsgegenstand. Was ist mit „Kultur“ gemeint? „Kultur“ wird als ein sich überlagerndes Gebilde von Phänomenen begriffen, welche mittels Mythen, Sprachbildern, „materiellen Bildern, ideologische[n] oder epistemische[n] Weltbildern“45 auf komplexe, sich überlagernde Wissenszusammenhänge verweisen. ${ }^{46}$ Diese kulturellen Wissensbestände durchlaufen kontinuierliche Umdeutungen und sind folglich von einem dynamischen Wandel geprägt. Darin liegt ihre paradox anmutende, ambivalente Eigenschaft aus Kontinuität und Diskontinuität begründet. ${ }^{47}$

2. „Wissen“ im Sinne der deutschsprachigen Wissensgeschichte kann als „Wissenskultur“ untersucht werden. Glückswissen wird in der vorliegenden Untersuchung „als Teil des kulturellen Wissens“48 begriffen. Wissen steht in Beziehung zu „(anderen) kulturellen Denk-, Orientierungs- und Handlungsmustern“ und kann unter einer Vielzahl von Akteur*innen, Institutionen und Praktiken, die „zueinander in vielfältigen, wandelbaren und oft weite Distanzen überwindenden Austausch- [...] und Machtbeziehungen“ stehen, untersucht werden. ${ }^{49}$ Unter dieser Prämisse fragt die Untersuchung nach Denkmustern, Metaphern ${ }^{50}$ und Begrifflichkeiten, mit welchen zu verschiedenen Zeitpunkten in unterschiedlichen Ländern Wissen über „Glück“ von verschiedenen Akteur*innen und sozialen Gruppierungen negiert, verändert, verbreitet und rezipiert wurde.

In Erweiterung des deutschsprachigen, wissenshistorischen Ansatzes eines „an bestimmte Kulturen gebunde[nen] “51 Wissensbegriffs sollen eine Vielzahl ambivalenter ,Glückskulturen ${ }^{\star}$ als Wissenskulturen erforscht werden.

44 Vgl. Hitler 2016b, 499.

45 Wirth 2008, 46.

46 Vgl. u. a. Baecker 2001.

47 Denkanstoß für diese These vgl. Kundrus und Steinbacher 2013.

48 Vgl. Müller-Wille, Reinhardt, Sommer 2017, 4.

49 Vgl. ebd. 4, 10.

$50 \mathrm{Zu}$ Metaphern in der Wissensgeschichte, vgl. Rouse 1993.

51 Vgl. Müller-Wille, Reinhardt, Sommer 2017, 3. 
,Glückskulturen' werden im Folgenden als ein Beziehungsgeflecht von Bedeutungszusammenhängen und Machtbeziehungen aufgefasst. Diese wissenshistorischen Bedeutungen sind wiederum an verschiedene Handlungsintentionen und -mechanismen geknüpft, welche an unterschiedlichen Orten kulturspezifische Vorstellungen von Glückswissen hervorbringen und austauschen.

3. „Politik“, oder vielmehr das „Politische ${ }^{\text {“52 }}$, als ein kulturwissenschaftlicher Untersuchungsgegenstand, durchläuft, vergleichbar mit „Kultur“ und „Wissen“, einen dynamischen und historisch komplexen Bedeutungswandel. In Anlehnung an den Sammelband von Uffa Jensen und Maik Tändler zu „Psychowissen und Politik im 20. Jahrhundert“ geht die vorliegende Untersuchung von einem Untersuchungsfeld politischer Machtbeziehungen aus, das „Politik“ als „Prozesse der Entstehung, Legimitierung, Vermittlung, Verfestigung und Erosion von Macht- und Herrschaftsverhältnissen“ analysiert. ${ }^{53}$ Folglich nimmt die Untersuchung „Praktiken symbolisch-kommunikativer Sinnstiftung“"54 in den Fokus und fragt nach den wissenschafts-, gefühls-, kultur-, geschlechter- und semantikgeschichtlichen Kontexten, in denen wissenspolitische Deutungsmuster im Austausch vielschichtiger Machtbeziehungen (re-)produziert, zurückgewiesen und eingegrenzt wurden.

52 Zum wissenshistorischen Begriff des Politischen im Entstehungskontext der Politikwissenschaft zur Zeit der Weimarer Republik vgl. Gangl 2008.

53 Vgl. Tändler und Jensen 2012, 14.

54 Vgl. ebd., 14. 